\title{
Walsogynes $\mathrm{H}-\mathrm{O}$ from Walsura chrysogyne
}

\author{
Alfarius Eko Nugroho ${ }^{1}$. Saori Nakajima ${ }^{1}$. Chin Piow Wong ${ }^{1}$. Yusuke Hirasawa ${ }^{1} \cdot$ Toshio Kaneda $^{1} \cdot$ Osamu Shirota ${ }^{2}$. \\ Takahiro Tougan $^{3} \cdot$ Toshihiro Horii $^{4} \cdot$ A. Hamid A. Hadi ${ }^{5} \cdot$ Hiroshi Morita $^{1}$
}

Received: 21 June 2021 / Accepted: 29 July 2021 / Published online: 5 August 2021

(C) The Japanese Society of Pharmacognosy 2021

\begin{abstract}
Eight new limonoids, walsogynes $\mathrm{H}-\mathrm{O}(\mathbf{1 - 8})$ were isolated from the barks of Walsura chrysogyne, and their structures were determined on the basis of the 1D and 2D NMR data. Walsogynes H-M (1-6) and O (8) were concluded to be 11,12-seco limonoids with a dodecahydro- $1 H$-naphtho[1,8-bc:3,4-c']difuran skeleton, and walsogyne $\mathrm{N}(7)$ to be 11,12-seco limonoid sharing a unique dodecahydronaphtho[1,8-bc:5,4- $\left.b^{\prime} c^{\prime}\right]$ difuran skeleton. Walsogynes $\mathrm{H}-\mathrm{O}(\mathbf{1}-\mathbf{8})$ exhibited potent antimalarial activity against Plasmodium falciparum $3 \mathrm{D} 7$ strain with $\mathrm{IC}_{50}$ value of $2.5,2.6,1.6,2.5,1.5,2.6,2.1$, and $1.1 \mu \mathrm{M}$, respectively.
\end{abstract}

\section{Graphic abstract}

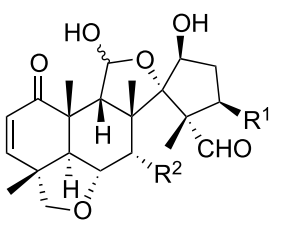

walsogyne $\mathrm{H}$ : $\alpha-\mathrm{OH} ; \mathrm{R}^{1}=\mathrm{A} ; \mathrm{R}^{2}=\mathrm{C}$ walsogyne $\mathrm{J}: \alpha-\mathrm{OH} ; \mathrm{R}^{1}=\mathbf{A} ; \mathrm{R}^{2}=\mathbf{D}$ walsogyne $\mathrm{K}: \beta-\mathrm{OH} ; \mathrm{R}^{1}=\mathbf{A} ; \mathrm{R}^{2}=\mathbf{C}$ walsogyne $L: \beta-O H ; R^{1}=A ; R^{2}=D$ walsogyne $O$ : $\beta-O H ; R^{1}=B ; R^{2}=C$

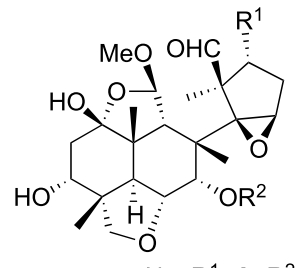

walsogyne $\mathrm{N}: \mathrm{R}^{1}=\mathrm{A} ; \mathrm{R}^{2}=\mathrm{C}$

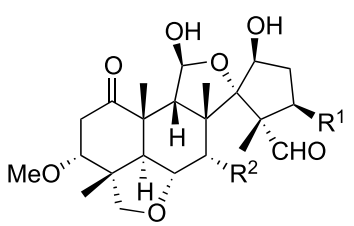

walsogyne I $: R^{1}=A ; R^{2}=C$ walsogyne $M: R^{1}=B ; R^{2}=C$

Keywords Limonoids - Walsura chrysogyne - Antimalarial activity

Hiroshi Morita

moritah@hoshi.ac.jp

1 Faculty of Pharmaceutical Sciences, Hoshi University, Ebara 2-4-41 Shinagawa-ku, Tokyo 142-8501, Japan

2 Faculty of Pharmaceutical Sciences at Kagawa Campus, Tokushima Bunri University, 1314-1 Shido, Sanuki City, Kagawa 769-2193, Japan

3 Research Center for Infectious Disease Control, Research Institute for Microbial Diseases, Osaka University, 3-1 Yamadaoka, Suita, Osaka 565-0871, Japan
4 Department of Malaria Vaccine Development, Research Institute for Microbial Diseases, Osaka University, 3-1 Yamadaoka, Suita, Osaka 565-0871, Japan

5 Department of Chemistry, Faculty of Science, University of Malaya, 50603 Kuala Lumpur, Malaysia 


\section{Introduction}

Walsura, a genus belonging to Meliaceae, is composed of about 16 species distributed from Sri Lanka to the Himalaya and Indochina through Malaysia, Indonesia to New Guinea, and Walsura chrysogyne is distributed in Peninsular Malaysia [1]. The plants of this genus have been reported to produce limonoids, dammarane, tirucallane and apotirucallane triterpenoids [2-7]. In our search for new bioactive compounds [8-27], we have reported the isolation of new limonoids and triterpenoids from plants of this genus $[3,4,19]$, and alkaloids showing antimalarial activity [22, $26,27]$. Continuation of the bioassay-guided investigation on the extract of $W$. chrysogyne bark led to the isolation of eight new limonoids, walsogynes $\mathrm{H}-\mathrm{O}$ (1-8) showing antimalarial activity (Fig. 1). Structure elucidation of 1-8 and their antimalarial activity of a series of walsogynes are reported herein.

\section{Results and discussions}

Compounds 1-8 were obtained as optically active white amorphous solids. The ${ }^{1} \mathrm{H}$ and ${ }^{13} \mathrm{C}$ NMR data (Tables 1 and 2) suggested the identity of $\mathbf{1 - 8}$ as walsogynes derivatives. Based on the ${ }^{13} \mathrm{C}$ NMR data, 1-6 and $\mathbf{8}$ were assumed to be derivatives of walsogyne $\mathrm{A}$ [2], and 7 was assumed to be a derivative of walsogyne $\mathrm{B}$ [4].

Compounds $\mathbf{1}$ and $\mathbf{4}$ were determined to have the same molecular formula, $\mathrm{C}_{31} \mathrm{H}_{38} \mathrm{O}_{11}$, based on the HR-ESIMS
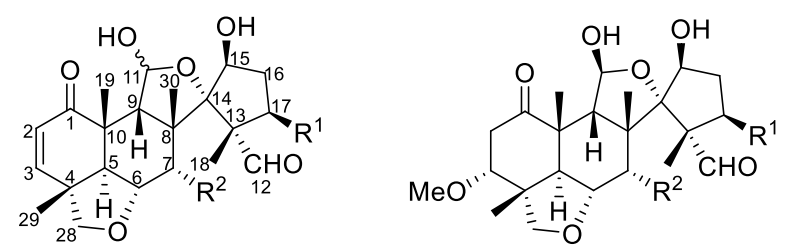

$1: 11 \alpha-O H ; R^{1}=A ; R^{2}=C$

3: $11 \alpha-O H ; R^{1}=A ; R^{2}=\mathbf{D}$

$4: 11 \beta-O H ; R^{1}=A ; R^{2}=\mathbf{C}$

$5: 11 \beta-\mathrm{OH} ; \mathrm{R}^{1}=\mathbf{A} ; \mathrm{R}^{2}=\mathbf{D}$

$8: 11 \beta-O H ; R^{1}=B ; R^{2}=C$

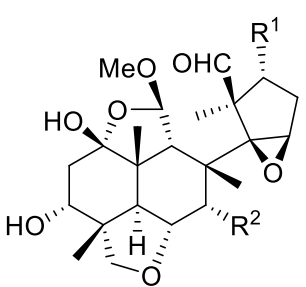

7: $R^{1}=A ; R^{2}=C$
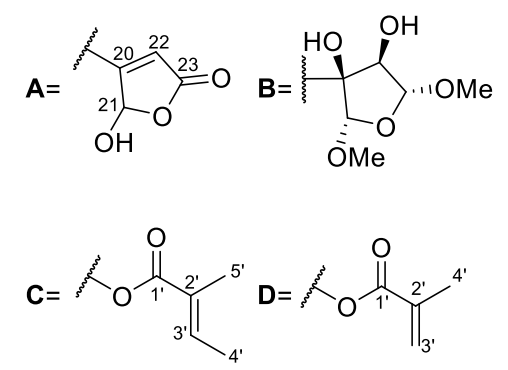

data. Their ${ }^{1} \mathrm{H}$ and ${ }^{13} \mathrm{C}$ NMR data are highly similar and the differences are similar to the differences of walsogynes $F$ and $G$ [4], indicating the structure of $\mathbf{1}$ as 11-epi4. Furthermore, except for the signal associated with the $\alpha, \beta$-unsaturated- $\gamma$-lactone moiety (C-20-C-23), the ${ }^{1} \mathrm{H}$ and

${ }^{13} \mathrm{C}$ NMR data of $\mathbf{4}$ and walsogyne A were highly similar. Thus, the structures of $\mathbf{1}$ and $\mathbf{4}$ were deduced to be as shown in Fig. 1. Analysis of the 2D NMR data further supported the proposed structures.

Compounds $\mathbf{3}$ and $\mathbf{5}$ were also determined to possess the same molecular formula, $\mathrm{C}_{30} \mathrm{H}_{36} \mathrm{O}_{11}$. Furthermore, their NMR data are highly similar to each other and to 1 and 4. However, the signals associated with $\mathrm{CH}-3^{\prime}$ and $\mathrm{CH}_{3}-4^{\prime}$ of the tiglate moiety in $\mathbf{1}$ and $\mathbf{4}$ are not observed in $\mathbf{3}$ and $\mathbf{5}$, and $\mathrm{sp}^{2}$ methylene signals $\left(\delta_{\mathrm{C}} 127.2, \delta_{\mathrm{H}} 5.61\right.$ and 6.49, and $\delta_{\mathrm{C}} 125.9, \delta_{\mathrm{H}} 5.67$ and 5.92 for $\mathbf{3}$ and $\mathbf{5}$ ) are observed instead. Therefore, 3 and $\mathbf{5}$ should have a methacrylate moiety instead of a tiglate moiety. Analysis of the 2D NMR data supported the structures of $\mathbf{3}$ and $\mathbf{5}$ to be as shown in Fig. 1. Specifically, the presence of a methacrylate moiety was supported by the HMBC correlations of $\mathrm{H}_{3}-4^{\prime}$ to $\mathrm{C}-1^{\prime}, \mathrm{C}-2^{\prime}$ and $\mathrm{C}-3^{\prime}$.

Compound 2 was revealed to have the molecular formula $\mathrm{C}_{32} \mathrm{H}_{42} \mathrm{O}_{12}$ by HRESITOFMS. Its NMR data are highly similar to 4 . However, the signals for $\mathrm{H}-2$ and $\mathrm{H}-3$ in $\mathbf{1}$ are not observed in $\mathbf{2}$, and a methoxy signal $\left(\delta_{\mathrm{H}} 3.34\right)$ and three aliphatic signals $\left(\delta_{\mathrm{H}} 2.66, \mathrm{~d}, 17.5 ; \delta_{\mathrm{H}} 3.14, \mathrm{~d}\right.$, 17.5; and $\delta_{\mathrm{H}} 3.58$, br s) are observed instead. Based on the chemical shifts and the multiplicity patterns, 2 was proposed to be 2,3-dihydro-3 $\alpha$-methoxy-4. The proposed structure was also confirmed through analysis of the $2 \mathrm{D}$ NMR data. In particular, the HMBC correlations of $\mathrm{H}_{3}-29$ and the methoxy to $\mathrm{C}-3$, and $\mathrm{H}-3$ to $\mathrm{C}-1$, and the NOESY correlation of $\mathrm{H}-3 / \mathrm{H}_{3}-29$ confirmed the position and the configuration of the methoxy at C-3.

By HRESITOFMS, $\mathbf{6}$ and $\mathbf{8}$ were revealed to have the molecular formula $\mathrm{C}_{34} \mathrm{H}_{50} \mathrm{O}_{14}$ and $\mathrm{C}_{33} \mathrm{H}_{46} \mathrm{O}_{13}$, respectively. Their NMR data are also highly similar to $\mathbf{4}$ and $\mathbf{2}$, respectively, differing only on the signals assigned to the $\alpha, \beta$-unsaturated- $\gamma$-lactone in $\mathbf{2}$ and $\mathbf{4}$. Analysis of the NMR data revealed that the furan moiety in both 6 and 8 were highly oxidized. The planar structure of the modified furan moiety in 6 was deduced from the ${ }^{1} \mathrm{H}-{ }^{1} \mathrm{H}$ COSY correlation of $\mathrm{H}-22$ and $\mathrm{H}-23$, and the $\mathrm{HMBC}$ correlations of $\mathrm{H}-21$ to $\mathrm{C}-17$ and $\mathrm{C}-20, \mathrm{H}-22$ to $\mathrm{C}-17, \mathrm{H}-23$ to $\mathrm{C}-21$, a methoxy $\left(\delta_{\mathrm{H}} 3.07\right)$ to $\mathrm{C}-21$ and a methoxy $\left(\delta_{\mathrm{H}} 3.38\right)$ to $\mathrm{C}-23$ (Fig. 2). The relative configuration of the furan moiety was then deduced from the ROESY correlations in pyridine- $d_{6}$ to be as shown in Fig. 3 since only the proposed configuration in Fig. 1 will fulfill the conditions set by the ROESY correlations shown in Fig. 3. Based on the ${ }^{1} \mathrm{H}$ and ${ }^{13} \mathrm{C}$ NMR data of $\mathbf{6}$ and $\mathbf{8}$, the furan moiety in $\mathbf{8}$

Fig. 1 Structures of $1-8$ 
Table $1{ }^{1} \mathrm{H}$ NMR data of $\mathbf{1 - 8}$ in $\mathrm{CD}_{3} \mathrm{OD}$ (* in DMSO-d6)

\begin{tabular}{|c|c|c|c|c|c|c|}
\hline & 1 & \multicolumn{2}{|l|}{2} & 3 & 4 & 5 \\
\hline $2 \mathrm{a}$ & $5.91(1 \mathrm{H}, \mathrm{d}, 9.8)$ & \multicolumn{2}{|c|}{$2.66(1 \mathrm{H}, \mathrm{d}, 17.5)$} & $5.90(1 \mathrm{H}, \mathrm{d}, 9.8)$ & $5.97(1 \mathrm{H}, \mathrm{d}, 9.8)$ & $5.96(1 \mathrm{H}, \mathrm{d}, 9.7)$ \\
\hline $2 b$ & & \multicolumn{3}{|c|}{$3.14(1 \mathrm{H}, \mathrm{d}, 17.5)$} & & \\
\hline 3 & $7.05(1 \mathrm{H}, \mathrm{d}, 9.8)$ & \multicolumn{2}{|c|}{$3.58(1 \mathrm{H}, \mathrm{brs})$} & $7.05(1 \mathrm{H}, \mathrm{d}, 9.8)$ & $7.20(1 \mathrm{H}, \mathrm{d}, 9.8)$ & $7.20(1 \mathrm{H}, \mathrm{d}, 9.8)$ \\
\hline 5 & $3.53(1 \mathrm{H}, \mathrm{d}, 12.9)$ & \multicolumn{2}{|c|}{$3.00(1 \mathrm{H}, \mathrm{d}, 12.5)$} & $3.53(1 \mathrm{H}, \mathrm{d}, 13.0)$ & $2.65(1 \mathrm{H}, \mathrm{d}, 12.6)$ & $2.65(1 \mathrm{H}, \mathrm{d}, 12.6)$ \\
\hline 6 & $4.38(1 \mathrm{H}, \mathrm{dd}, 12.9,3.2)$ & \multicolumn{2}{|c|}{$4.41(1 \mathrm{H}, \mathrm{d}, 12.5)$} & $4.40(1 \mathrm{H}, \mathrm{dd}, 13.2,3.0)$ & $4.46(1 \mathrm{H}, \mathrm{dd}, 12.6,2.9)$ & $4.47(1 \mathrm{H}, \mathrm{dd}, 12.7,2.7)$ \\
\hline 7 & $5.45(1 \mathrm{H}, \mathrm{d}, 3.2)$ & \multicolumn{2}{|c|}{$5.32(1 \mathrm{H}, \mathrm{brs})$} & $5.46(1 \mathrm{H}, \mathrm{d}, 3.0)$ & $5.37(1 \mathrm{H}, \mathrm{d}, 3.0)$ & $5.38(1 \mathrm{H}, \mathrm{d}, 2.9)$ \\
\hline 9 & $2.75(1 \mathrm{H}, \mathrm{d}, 6.7)$ & \multicolumn{2}{|c|}{$2.75(1 \mathrm{H}, \mathrm{d}, 6.4)$} & $2.76(1 \mathrm{H}, \mathrm{d}, 6.6)$ & $2.85(1 \mathrm{H}, \mathrm{d}, 7.1)$ & $2.84(1 \mathrm{H}, \mathrm{d}, 7.0)$ \\
\hline 11 & $5.51(1 \mathrm{H}, \mathrm{d}, 6.7)$ & \multicolumn{2}{|c|}{$5.24(1 \mathrm{H}, \mathrm{d}, 6.4)$} & $5.51(1 \mathrm{H}, \mathrm{d}, 6.6)$ & $5.27(1 \mathrm{H}, \mathrm{d}, 7.1)$ & $5.25(1 \mathrm{H}, \mathrm{d}, 7.1)$ \\
\hline 12 & $9.67(1 \mathrm{H}, \mathrm{s})$ & \multicolumn{2}{|c|}{$9.58(1 \mathrm{H}, \mathrm{s})$} & $9.72(1 \mathrm{H}, \mathrm{s})$ & $9.58(1 \mathrm{H}, \mathrm{s})$ & $9.64(1 \mathrm{H}, \mathrm{m})$ \\
\hline 15 & $4.31(1 \mathrm{H}, \mathrm{d}, 4.7)$ & 4.91( & $4.5)$ & $4.31(1 \mathrm{H}, \mathrm{d}, 4.8)$ & $4.91(1 \mathrm{H}, \mathrm{m})$ & $4.91(1 \mathrm{H}, \mathrm{d}, 5.3)$ \\
\hline $16 \mathrm{a}$ & $1.77(1 \mathrm{H}, \mathrm{m})$ & 1.79( & & $1.77(1 \mathrm{H}, \mathrm{m})$ & $1.82(1 \mathrm{H}, \mathrm{m})$ & $1.82(1 \mathrm{H}, \mathrm{m})$ \\
\hline $16 b$ & $\begin{array}{l}2.68(1 \mathrm{H}, \mathrm{ddd}, 16.3,11.1 \text {, } \\
5.3)\end{array}$ & 2.79( & & $2.68(1 \mathrm{H}, \mathrm{m})$ & $\begin{array}{l}2.78(1 \mathrm{H}, \mathrm{ddd}, 19.0,11.0, \\
5.4)\end{array}$ & $\begin{array}{l}2.77(1 \mathrm{H}, \mathrm{ddd}, 15.6,10.6 \text {, } \\
5.1)\end{array}$ \\
\hline 17 & $3.40(1 \mathrm{H}, \mathrm{m})$ & 3.48( & & $3.41(1 \mathrm{H}, \mathrm{m})$ & $3.48(1 \mathrm{H}, \mathrm{m})$ & $3.47(1 \mathrm{H}, \mathrm{m})$ \\
\hline 18 & $1.23(3 \mathrm{H}, \mathrm{s})$ & 1.22( & & $1.23(3 \mathrm{H}, \mathrm{s})$ & $1.22(3 \mathrm{H}, \mathrm{s})$ & $1.23(3 \mathrm{H}, \mathrm{m})$ \\
\hline 19 & $1.33(3 \mathrm{H}, \mathrm{s})$ & 1.43( & & $1.33(3 \mathrm{H}, \mathrm{s})$ & $1.40(3 \mathrm{H}, \mathrm{s})$ & $1.38(3 \mathrm{H}, \mathrm{m})$ \\
\hline 21 & $5.45^{*}(1 \mathrm{H}, \mathrm{brs})$ & $5.37 *$ & & $5.34 *(1 \mathrm{H}, \mathrm{m})$ & $5.45^{*}(1 \mathrm{H}, \mathrm{m})$ & $5.45^{*}(1 \mathrm{H}, \mathrm{m})$ \\
\hline 22 & $6.07 *(1 \mathrm{H}, \mathrm{brs})$ & $6.13 *$ & & $6.28^{*}(1 \mathrm{H}, \mathrm{m})$ & $6.25^{*}(1 \mathrm{H}, \mathrm{m})$ & $6.26^{*}(1 \mathrm{H}, \mathrm{m})$ \\
\hline $28 \mathrm{a}$ & $3.27(1 \mathrm{H}, \mathrm{m})$ & 3.44( & $7.0)$ & $3.31(1 \mathrm{H}, \mathrm{m})$ & $3.30(1 \mathrm{H}, \mathrm{d}, 7.2)$ & $3.34(1 \mathrm{H}, \mathrm{m})$ \\
\hline $28 b$ & $3.60(1 \mathrm{H}, \mathrm{d}, 6.9)$ & 3.67( & $7.0)$ & $3.61(1 \mathrm{H}, \mathrm{d}, 7.0)$ & $3.67(1 \mathrm{H}, \mathrm{d}, 7.2)$ & $3.69(1 \mathrm{H}, \mathrm{d}, 7.3)$ \\
\hline 29 & $1.25(3 \mathrm{H}, \mathrm{s})$ & 1.32( & & $1.25(3 \mathrm{H}, \mathrm{s})$ & $1.29(3 \mathrm{H}, \mathrm{s})$ & $1.29(3 \mathrm{H}, \mathrm{m})$ \\
\hline 30 & $1.66(3 \mathrm{H}, \mathrm{s})$ & 1.64( & & $1.66(3 \mathrm{H}, \mathrm{s})$ & $1.67(3 \mathrm{H}, \mathrm{s})$ & $1.67(3 \mathrm{H}, \mathrm{m})$ \\
\hline 3'a & $5.61(1 \mathrm{H}, \mathrm{qd}, 7.1,1.3$ & 6.79( & $6.5)$ & $5.61(1 \mathrm{H}, \mathrm{s})$ & $6.72(1 \mathrm{H}, \mathrm{q}, 6.4)$ & $5.67(1 \mathrm{H}, \mathrm{m})$ \\
\hline $3^{\prime} \mathrm{b}$ & & & & $6.49(1 \mathrm{H}, \mathrm{s})$ & & $5.92(1 \mathrm{H}, \mathrm{m})$ \\
\hline $4^{\prime}$ & $1.76(3 \mathrm{H}, \mathrm{d}, 7.1)$ & 1.84( & $6.5)$ & $1.89(3 \mathrm{H}, \mathrm{m})$ & $1.81(3 \mathrm{H}, \mathrm{d}, 6.4)$ & \\
\hline $5^{\prime}$ & $1.79(3 \mathrm{H}, \mathrm{brs})$ & 1.84( & & & $1.82(3 \mathrm{H}, \mathrm{s})$ & $1.92(3 \mathrm{H}, \mathrm{m})$ \\
\hline 3-OMe & & 3.34( & & & & \\
\hline & 6 & & & 7 & 7 & 8 \\
\hline & In $\mathrm{CD}_{3} \mathrm{OD}$ & & In $\mathrm{C}_{5} \mathrm{D}$ & & In DMSO- $d_{6}$ & In $\mathrm{CD}_{3} \mathrm{OD}$ \\
\hline $2 \mathrm{a}$ & $2.62(1 \mathrm{H}, \mathrm{d}, 12.6)$ & & $2.71(1$ & $\mathrm{H}, \mathrm{d}, 17.7)$ & $1.92(1 \mathrm{H}, \mathrm{m})$ & $5.95(1 \mathrm{H}, \mathrm{d}, 9.8)$ \\
\hline $2 b$ & $2.62(1 \mathrm{H}, \mathrm{d}, 12.6)$ & & $3.12(1$ & $\mathrm{H}, \mathrm{d}, 17.7)$ & $1.98(1 \mathrm{H}, \mathrm{m})$ & \\
\hline 3 & $3.57(1 \mathrm{H}, \mathrm{m})$ & & $3.43(1$ & H, brs) & $3.57(1 \mathrm{H}, \mathrm{m})$ & $7.18(1 \mathrm{H}, \mathrm{d}, 9.8)$ \\
\hline 5 & $2.97(1 \mathrm{H}, \mathrm{d}, 12.4)$ & & $3.37(1$ & $\mathrm{H}, \mathrm{d}, 12.2)$ & $2.44(1 \mathrm{H}, \mathrm{d}, 12.8)$ & $2.63(1 \mathrm{H}, \mathrm{d}, 12.5)$ \\
\hline 6 & $4.40(1 \mathrm{H}, \mathrm{dd}, 12.4,2.7$ & & $4.57(1$ & $\mathrm{H}, \mathrm{dd}, 12.2,2.8)$ & $4.11(1 \mathrm{H}, \mathrm{dd}, 12.6,2.9)$ & $4.44(1 \mathrm{H}, \mathrm{dd}, 12.5,3.0)$ \\
\hline 7 & $5.26(1 \mathrm{H}, \mathrm{d}, 2.7)$ & & $5.87(1$ & $\mathrm{H}, \mathrm{d}, 2.8)$ & $5.18(1 \mathrm{H}, \mathrm{d}, 2.9)$ & $5.32(1 \mathrm{H}, \mathrm{d}, 3.0)$ \\
\hline 9 & $2.72(1 \mathrm{H}, \mathrm{d}, 6.5)$ & & $3.23(1$ & $\mathrm{H}, \mathrm{d}, 6.6)$ & $2.41(1 \mathrm{H}, \mathrm{m})$ & $2.82(1 \mathrm{H}, \mathrm{d}, 7.1)$ \\
\hline 11 & $5.18(1 \mathrm{H}, \mathrm{d}, 6.5)$ & & $5.81(1$ & $\mathrm{H}, \mathrm{dd}, 6.6,2.4)$ & $5.31(1 \mathrm{H}, \mathrm{d}, 5.8)$ & $5.21(1 \mathrm{H}, \mathrm{d}, 7.1)$ \\
\hline 12 & $9.31(1 \mathrm{H}, \mathrm{s})$ & & 10.03 & $1 \mathrm{H}, \mathrm{s})$ & $9.76(1 \mathrm{H}, \mathrm{m})$ & $9.32(1 \mathrm{H}, \mathrm{m})$ \\
\hline 15 & $4.82(1 \mathrm{H}, \mathrm{m})$ & & $5.55(1$ & $\mathrm{H}, \mathrm{m})$ & $3.58(1 \mathrm{H}, \mathrm{brs})$ & $4.82(1 \mathrm{H}, \mathrm{m})$ \\
\hline $16 \mathrm{a}$ & $2.11(1 \mathrm{H}, \mathrm{m})$ & & $3.01(1$ & $\mathrm{H}, \mathrm{m})$ & $1.97(1 \mathrm{H}, \mathrm{m})$ & $2.10(1 \mathrm{H}, \mathrm{m})$ \\
\hline $16 b$ & $2.54(1 \mathrm{H}, \mathrm{ddd}, 16.5,10$ & $5,5.7)$ & $3.16(1$ & $\mathrm{H}, \mathrm{m})$ & $2.19(1 \mathrm{H}, \mathrm{m})$ & $2.54(1 \mathrm{H}, \mathrm{ddd}, 16.6,10.8,5.7)$ \\
\hline 17 & $2.93(1 \mathrm{H}, \mathrm{m})$ & & $3.57(1$ & $\mathrm{H}, \mathrm{m})$ & $2.77(1 \mathrm{H}, \mathrm{d}, 8.0)$ & $2.93(1 \mathrm{H}, \mathrm{m})$ \\
\hline 18 & $1.43(3 \mathrm{H}, \mathrm{s})$ & & $2.09(3$ & $\mathrm{H}, \mathrm{s})$ & $1.05(3 \mathrm{H}, \mathrm{m})$ & $1.43(3 \mathrm{H}, \mathrm{s})$ \\
\hline 19 & $1.42(3 \mathrm{H}, \mathrm{s})$ & & $1.33(3$ & $\mathrm{H}, \mathrm{s})$ & $1.05(3 \mathrm{H}, \mathrm{m})$ & $1.38(3 \mathrm{H}, \mathrm{s})$ \\
\hline 21 & $4.54(1 \mathrm{H}, \mathrm{s})$ & & $5.24(1$ & $\mathrm{H}, \mathrm{s})$ & $5.59(1 \mathrm{H}, \mathrm{m})$ & $4.51(1 \mathrm{H}, \mathrm{m})$ \\
\hline 22 & $3.85(1 \mathrm{H}, \mathrm{d}, 3.6)$ & & $4.55(1$ & $\mathrm{H}, \mathrm{dd}, 7.3,3.7)$ & $6.25(1 \mathrm{H}, \mathrm{m})$ & $3.84(1 \mathrm{H}, \mathrm{d}, 3.6)$ \\
\hline 23 & $4.72(1 \mathrm{H}, \mathrm{d}, 3.6)$ & & $5.28(1$ & $\mathrm{H}, \mathrm{d}, 3.7)$ & & $4.72(1 \mathrm{H}, \mathrm{d}, 3.6)$ \\
\hline $28 \mathrm{a}$ & $3.43(1 \mathrm{H}, \mathrm{d}, 6.5)$ & & $3.58(1$ & $\mathrm{H}, \mathrm{d}, 6.7)$ & $3.22(1 \mathrm{H}, \mathrm{m})$ & $3.29(1 \mathrm{H}, \mathrm{m})$ \\
\hline $28 b$ & $3.65(1 \mathrm{H}, \mathrm{d}, 6.5)$ & & $3.92(1$ & $\mathrm{H}, \mathrm{d}, 6.7)$ & $3.62(1 \mathrm{H}, \mathrm{m})$ & $3.66(1 \mathrm{H}, \mathrm{d}, 7.2)$ \\
\hline
\end{tabular}


Table 1 (continued)

\begin{tabular}{lllll}
\hline & $\mathbf{6}$ & & $\mathbf{7}$ & $\mathbf{8}$ \\
\hline 29 & $1.31(3 \mathrm{H}, \mathrm{s})$ & $1.25(3 \mathrm{H}, \mathrm{s})$ & $1.32(3 \mathrm{H}, \mathrm{m})$ & $1.28(3 \mathrm{H}, \mathrm{s})$ \\
30 & $1.60(3 \mathrm{H}, \mathrm{s})$ & $1.86(3 \mathrm{H}, \mathrm{s})$ & $0.94(3 \mathrm{H}, \mathrm{m})$ & $1.64(3 \mathrm{H}, \mathrm{s})$ \\
$3^{\prime}$ & $6.75(1 \mathrm{H}, \mathrm{q}, 7.0)$ & $7.03(1 \mathrm{H}, \mathrm{q}, 9.7)$ & $6.75(1 \mathrm{H}, \mathrm{q}, 7.1)$ & $6.69(1 \mathrm{H}, \mathrm{q}, 7.0)$ \\
$4^{\prime}$ & $1.84(3 \mathrm{H}, \mathrm{d}, 7.0)$ & $1.65(3 \mathrm{H}, \mathrm{d}, 7.1)$ & $1.78(3 \mathrm{H}, \mathrm{d}, 7.1)$ & $1.83(3 \mathrm{H}, \mathrm{d}, 7.0)$ \\
$5^{\prime}$ & $1.82(3 \mathrm{H}, \mathrm{s})$ & $2.10(3 \mathrm{H}, \mathrm{s})$ & $1.82(3 \mathrm{H}, \mathrm{s})$ & $1.80(3 \mathrm{H}, \mathrm{s})$ \\
$3-\mathrm{OMe}$ & $3.32(3 \mathrm{H}, \mathrm{s})$ & $3.15(3 \mathrm{H}, \mathrm{s})$ & $3.38(3 \mathrm{H}, \mathrm{s})$ & $3.06(3 \mathrm{H}, \mathrm{s})$ \\
$11-\mathrm{OMe}$ & & & & $3.38(3 \mathrm{H}, \mathrm{s})$ \\
$21-\mathrm{OMe}$ & $3.07(3 \mathrm{H}, \mathrm{s})$ & $3.33(3 \mathrm{H}, \mathrm{s})$ & & \\
$23-\mathrm{OMe}$ & $3.38(3 \mathrm{H}, \mathrm{s})$ & $3.39(3 \mathrm{H}, \mathrm{s})$ & & \\
\hline
\end{tabular}

Table $2{ }^{13} \mathrm{C}$ NMR data of $\mathbf{1 - 8}$

\begin{tabular}{|c|c|c|c|c|c|c|c|c|c|}
\hline & $\mathbf{1}^{\mathrm{a}}$ & $2^{\mathrm{a}}$ & $3^{\mathrm{a}}$ & $4^{\mathrm{a}}$ & $5^{\mathrm{a}}$ & $6^{\mathrm{a}}$ & $6^{\mathrm{b}}$ & $7^{\mathrm{c}}$ & $8^{\mathrm{a}}$ \\
\hline 1 & 205.4 & 213.7 & 205.4 & 202.6 & 202.5 & 214.1 & 212.3 & 106.7 & 203.0 \\
\hline 2 & 131.1 & 40.4 & 131.1 & 131.2 & 131.2 & 40.2 & 40.0 & 37.2 & 131.0 \\
\hline 3 & 152.8 & 81.8 & 152.8 & 154.6 & 154.6 & 81.9 & 80.6 & 69.2 & 155.0 \\
\hline 4 & 42.6 & 44.5 & 42.7 & 42.7 & 42.7 & 44.5 & 43.4 & 42.5 & 42.6 \\
\hline 5 & 46.2 & 42.1 & 46.3 & 47.1 & 47.1 & 42.0 & 40.8 & 40.0 & 46.9 \\
\hline 6 & 74.7 & 73.9 & 74.6 & 74.4 & 74.4 & 74.1 & 73.0 & 71.6 & 74.6 \\
\hline 7 & 73.0 & 74.1 & 73.3 & 73.5 & 73.8 & 73.9 & 72.7 & 74.4 & 73.4 \\
\hline 8 & 52.8 & 56.5 & 52.8 & 56.5 & 56.6 & 56.7 & 55.8 & 43.5 & 56.8 \\
\hline 9 & 59.2 & 65.0 & 59.3 & 63.6 & 63.7 & 64.7 & 64.2 & 58.4 & 63.3 \\
\hline 10 & 46.1 & 50.3 & 46.2 & 46.3 & 46.3 & 50.3 & 49.2 & 46.8 & 46.3 \\
\hline 11 & 96.6 & 99.4 & 96.6 & 98.8 & 98.8 & 99.1 & 98.5 & 106.5 & 98.5 \\
\hline 12 & 202.6 & 201.3 & 203.0 & 201.6 & 201.9 & 202.9 & 200.9 & N.D & 203.0 \\
\hline 13 & 59.6 & 60.2 & 59.7 & 60.1 & 60.2 & 60.2 & 59.5 & 58.5 & 60.2 \\
\hline 14 & 98.4 & 100.3 & 98.5 & 99.9 & 100.0 & 100.7 & 100.0 & 72.0 & 100.0 \\
\hline 15 & 80.7 & 80.6 & 80.7 & 80.8 & 80.8 & 80.2 & 79.6 & 60.2 & 80.4 \\
\hline 16 & 40.2 & 39.4 & 40.2 & 39.4 & 39.5 & 33.1 & 33.0 & 29.5 & 33.2 \\
\hline 17 & 41.3 & 41.2 & 42.1 & 41.2 & 41.2 & 46.8 & 46.4 & N.D & 46.8 \\
\hline 18 & 12.7 & 13.1 & 12.8 & 13.1 & 13.1 & 14.2 & 14.3 & 13.0 & 14.3 \\
\hline 19 & 22.3 & 22.3 & 22.3 & 22.4 & 22.4 & 22.2 & 21.6 & 18.3 & 22.5 \\
\hline 20 & 148.6 & 148.6 & 148.6 & 148.6 & 148.6 & 82.6 & N.D & 148.6 & 82.5 \\
\hline 21 & 98.9 & 98.9 & 98.9 & 98.9 & 98.9 & 109.0 & 108.4 & 98.9 & 109.0 \\
\hline 22 & 120.4 & 120.4 & 120.4 & 120.4 & 120.4 & 82.5 & 81.8 & 120.4 & 82.6 \\
\hline 23 & 171.5 & 171.5 & 171.5 & 171.5 & 171.5 & 111.7 & 111.3 & 171.5 & 112.0 \\
\hline 28 & 80.7 & 77.7 & 80.7 & 80.4 & 80.4 & 77.5 & 76.4 & 76.8 & 80.3 \\
\hline 29 & 20.3 & 19.3 & 20.4 & 20.4 & 20.5 & 19.3 & 18.6 & 18.9 & 20.4 \\
\hline 30 & 25.8 & 24.1 & 25.8 & 23.8 & 23.9 & 23.6 & 23.7 & 23.9 & 23.4 \\
\hline $1^{\prime}$ & 169.2 & 168.4 & 168.6 & 168.0 & 167.4 & 168.4 & 166.4 & 164.9 & 168.0 \\
\hline $2^{\prime}$ & 130.8 & 130.7 & 138.8 & 130.9 & 138.9 & 130.8 & 130.5 & 128.1 & 131.0 \\
\hline $3^{\prime}$ & 139.7 & 139.0 & 127.2 & 138.6 & 125.9 & 138.3 & 135.9 & 137.4 & 138.0 \\
\hline $4^{\prime}$ & 14.5 & 12.1 & 18.4 & 14.6 & 18.5 & 12.1 & 14.1 & 14.4 & 12.3 \\
\hline $5^{\prime}$ & 12.0 & 14.6 & & 12.3 & & 14.6 & 14.3 & 12.3 & 14.6 \\
\hline 3-OMe & & 58.1 & & & & 58.1 & 57.5 & & \\
\hline 11-OMe & & & & & & & & 56.1 & \\
\hline 21-OMe & & & & & & 54.3 & 54.1 & & 54.3 \\
\hline 23-OMe & & & & & & 56.4 & 55.6 & & 56.4 \\
\hline
\end{tabular}

${ }^{\mathrm{a}}$ In $\mathrm{CD}_{3} \mathrm{OD} ;{ }^{\mathrm{b}}$ in $\mathrm{C}_{5} \mathrm{D}_{5} \mathrm{~N}$; ${ }^{\text {in }}$ DMSO- $d_{6}$ 


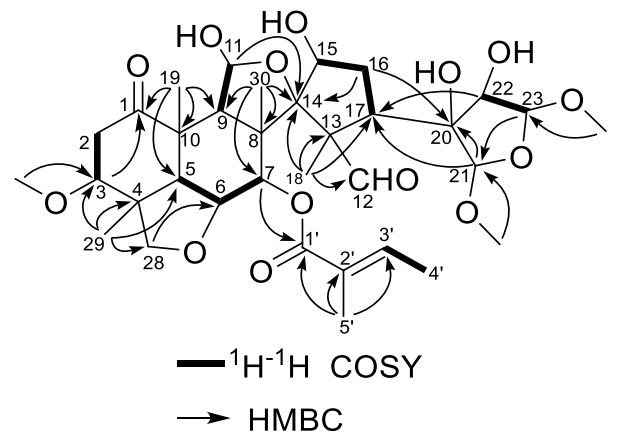

Fig. 2 Selected 2D NMR correlations of 6

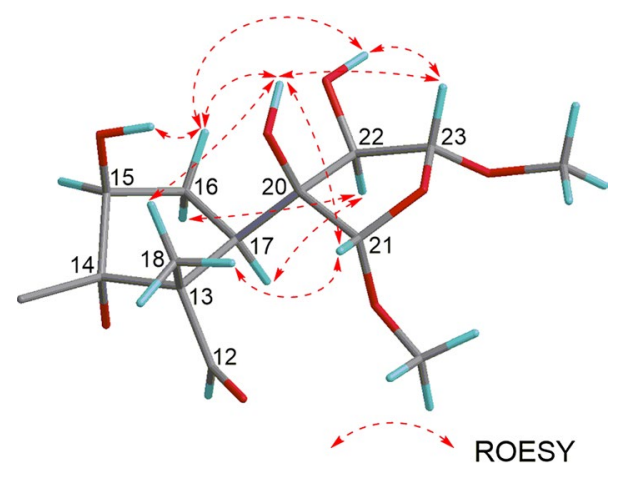

Fig. 3 Selected ROESY correlations of 6

should have the same relative configuration as in $\mathbf{6}$. Thus, the structure of $\mathbf{8}$ was proposed to be as shown in Fig. 1 .

Compound 7 was revealed to have the molecular formula $\mathrm{C}_{32} \mathrm{H}_{42} \mathrm{O}_{12}$ by HRESITOFMS. Its ${ }^{1} \mathrm{H}$ and ${ }^{13} \mathrm{C}$ NMR data are highly similar to walsogyne B. However, the NMR data suggested that the furan moiety in walsogyne B was oxidized to a lactone moiety similar to the one found in $\mathbf{1}$. Furthermore, the signals for $\mathrm{H}-2$ and $\mathrm{H}-3$ in walsogyne $\mathrm{B}$ are also not observed in 7 , and three aliphatic signals $\left(\delta_{\mathrm{H}} 1.92, \delta_{\mathrm{H}} 1.98\right.$ and $\left.\delta_{\mathrm{H}} 3.57\right)$ are observed instead. Finally, the HMBC correlations of $\mathrm{H}_{3}-29$ to $\mathrm{C}-3$ and $\mathrm{H}-3$ to $\mathrm{C}-1$ and the NOESY correlation of $\mathrm{H}-3 / \mathrm{H}_{3}-29$ confirmed the position and the $\alpha$ orientation of the hydroxy at C-3.

Considering that 1-8 were isolated from the same extract as walsogynes B-G [4], their absolute configurations were assumed to be similar to walsogynes B-G based on the biogenetic relationships.

\section{Antimalarial activity}

Walsogynes $\mathrm{H}-\mathrm{O}(\mathbf{1}-\mathbf{8})$ were tested for the antimalarial activity against Plasmodium falciparum 3D7 strain. The assay showed that 1-8 had potent in vitro antimalarial activity [the half-maximal (50\%) inhibitory concentration
$\left(\mathrm{IC}_{50}\right)=2.5,2.6,1.6,2.5,1.5,2.6,2.1$, and $1.1 \mu \mathrm{M}$, respectively] (Table 3).

From Walsura spp., one of limonoid peroxide has been reported to show antimalarial activity [28]. We also reported some limonoids, ceramicines A-D isolated from the barks of $C$. ceramicus, exhibited antimalarial activity against $P$. falciparum 3D7 in vitro [29]. However, the skeleton of these limonoids was different from that of walsogynes. A series of walsogynes $\mathrm{H}-\mathrm{O}(\mathbf{1 - 8})$ and walsogynes $\mathrm{B}, \mathrm{D}$, and $\mathrm{E}\left(\mathrm{IC}_{50}=2.4,2.6\right.$, and $2.6 \mu \mathrm{M}$, respectively) had more potent antimalarial activity than these known limonoids (Table 3). The activity might be depending on their unique 11,12-seco limonoid skeleton but not influenced by their substituent patterns.

\section{Experimental section}

\section{General experimental procedures}

Optical rotations were measured on a JASCO DIP-1000 polarimeter. UV spectra were recorded on a Shimadzu UVmini-1240 spectrophotometer and IR spectra on a JASCO FT/IR-4100 spectrophotometer. High-resolution ESI MS were obtained on a JMS-T100LP (JEOL). ${ }^{1} \mathrm{H}$ and 2D NMR spectra were measured on a $400 \mathrm{MHz}$ or $600 \mathrm{MHz}$ spectrometer at $300 \mathrm{~K}$, while ${ }^{13} \mathrm{C}$ NMR spectra were on a $100 \mathrm{MHz}$ or $150 \mathrm{MHz}$ spectrometer. The residual solvent peaks were used as internal standards $\left(\delta_{\mathrm{H}} 7.26\right.$ and $\delta_{\mathrm{C}} 77.0$ for $\mathrm{CDCl}_{3}, \delta_{\mathrm{H}} 3.31$ and $\delta_{\mathrm{C}} 49.0$ for $\mathrm{CD}_{3} \mathrm{OD}$ ).

\section{Material}

The barks of W. chrysogyne were collected in Mersing, Malaysia in October 2000. The botanical identification was made by Mr. Teo Leong Eng, Faculty of Science, University of Malaya. Voucher specimens (Herbarium No. 4957)

Table 3 Antimalarial activity of 1-8 and walsogynes B, D, and E against Plasmodium falciparum 3D7 strain

\begin{tabular}{ll}
\hline $\mathrm{IC}_{50}(\mu \mathrm{M})$ & \\
\hline $\mathbf{1}$ & 2.5 \\
$\mathbf{2}$ & 2.6 \\
$\mathbf{3}$ & 1.6 \\
$\mathbf{4}$ & 2.5 \\
$\mathbf{5}$ & 1.5 \\
$\mathbf{6}$ & 2.6 \\
$\mathbf{7}$ & 2.1 \\
$\mathbf{8}$ & 1.1 \\
Walsogyne B & 2.4 \\
Walsogyne D & 2.6 \\
Walsogyne E & 2.6 \\
\hline
\end{tabular}


are deposited in the Herbarium of Chemistry Department, University of Malaya.

\section{Extraction and isolation}

The dried ground barks of $W$. chrysogyne (440 g) were extracted successively with $\mathrm{MeOH}$ and $54 \mathrm{~g}$ of extract were obtained. The total extract was successively partitioned with $n$-hexane, EtOAc, $n$-BuOH, and water. The EtOAcsoluble materials $(11.5 \mathrm{~g})$ were separated with a silica gel column $\left(\mathrm{CHCl}_{3} / \mathrm{MeOH}, 1: 0 \rightarrow 0: 1\right)$ to obtain 10 fractions (E-1 E-10). Fraction E-5 was further separated to five fractions (E-5-1 E-5-5) with an LH-20 column $\left(\mathrm{CHCl}_{3} /\right.$ $\mathrm{MeOH}, 1: 1)$. Fraction E-5-3 was further separated with a silica gel column $\left(\mathrm{CHCl}_{3} / \mathrm{MeOH}, 1: 0 \rightarrow 0: 1\right)$ to obtain 13 fractions (E-5-3-1 E-5-3-13). Fraction E-5-3-9 was further separated by HPLC (Shiseido C18 MGII, $\mathrm{H}_{2} \mathrm{O} / \mathrm{MeCN}$, 70:30) to obtain impure 1-5. Impure $\mathbf{1}$ and $\mathbf{3}-\mathbf{5}$ were purified using HPLC (Nacalai tesque Cholester, $\mathrm{H}_{2} \mathrm{O} / \mathrm{MeCN}, 80: 20$ ) to obtain pure $\mathbf{1}\left(1.3 \mathrm{mg}, 0.0003 \%, t_{\mathrm{R}} 70 \mathrm{~min}\right), \mathbf{3}(1.3 \mathrm{mg}$, $\left.0.0003 \%, t_{\mathrm{R}} 58 \mathrm{~min}\right), 4\left(0.5 \mathrm{mg}, 0.0001 \%, t_{\mathrm{R}} 46 \mathrm{~min}\right)$ and $5\left(1.8 \mathrm{mg}, 0.0004 \%, t_{\mathrm{R}} 38 \mathrm{~min}\right)$. In addition, impure 2 was purified using HPLC (Nacalai tesque Cholester, $\mathrm{H}_{2} \mathrm{O} / \mathrm{MeCN}$, $75: 25)$ to obtain pure 2 ( $\left.1.8 \mathrm{mg}, 0.0004 \%, t_{\mathrm{R}} 48 \mathrm{~min}\right)$. Fraction E-5-3-8 was further separated by HPLC (Shiseido C18 MGII, $\mathrm{H}_{2} \mathrm{O} / \mathrm{MeCN}, 70: 30$ ) to obtain 6 (1.3 mg, 0.0003\%, $\left.t_{\mathrm{R}} 26 \mathrm{~min}\right), 7\left(2.4 \mathrm{mg}, 0.0005 \%, t_{\mathrm{R}} 20 \mathrm{~min}\right), 8(0.5 \mathrm{mg}$, $\left.0.0001 \%, t_{\mathrm{R}} 22 \mathrm{~min}\right)$.

Walsogyne $H(\mathbf{1})$ white amorphous solid. $[\alpha]_{\mathrm{D}}{ }^{28}-28$ (c 1.0, MeOH). IR (film) $v_{\max } 3411,2928,1746,1710$, $1671 \mathrm{~cm}^{-1}$. UV (MeOH) $\lambda_{\max }(\log \varepsilon) 216(4.32) \mathrm{nm} . \mathrm{CD}$ $(\mathrm{MeOH}) \lambda_{\max }(\Delta \varepsilon) 205$ (12.50), 228 (-1.79), 250 (2.29), $300(-1.01) \mathrm{nm}$. ESIMS $m / z, 609(\mathrm{M}+\mathrm{Na})^{+}$. HRESIMS $m / z 609.2335$ [calcd for $\mathrm{C}_{31} \mathrm{H}_{38} \mathrm{O}_{11} \mathrm{Na}(\mathrm{M}+\mathrm{Na})^{+}: 609.2312$ ].

Walsogyne I (2) white amorphous solid. $[\alpha]_{\mathrm{D}}^{28}-10$ (c 1.0, MeOH). IR (film) $v_{\max } 3435,2928,1743,1721$, $1711 \mathrm{~cm}^{-1}$. UV (MeOH) $\lambda_{\max }(\log \varepsilon) 213(4.52) \mathrm{nm} . \mathrm{CD}$ $(\mathrm{MeOH}) \lambda_{\max }(\Delta \varepsilon) 206$ (4.13), $226(-2.08), 247$ (1.36), $297(0.25) \mathrm{nm}$. ESIMS $\mathrm{m} / \mathrm{z} 641(\mathrm{M}+\mathrm{Na})^{+}$. HRESIMS $\mathrm{m} / \mathrm{z}$ 641.2571 [calcd for $\mathrm{C}_{32} \mathrm{H}_{42} \mathrm{O}_{12} \mathrm{Na}(\mathrm{M}+\mathrm{Na})^{+}$: 641.2574].

Walsogyne $J(3)$ white amorphous solid. $[\alpha]_{\mathrm{D}}^{28}-40(c$ $0.41, \mathrm{MeOH}$ ). IR (film) $v_{\max } 3411,2920,1715,1670 \mathrm{~cm}^{-1}$. $\mathrm{UV}(\mathrm{MeOH}) \lambda_{\max }(\log \varepsilon) 211(4.04) \mathrm{nm} . \mathrm{CD}(\mathrm{MeOH}) \lambda_{\max }$ ( $\Delta \varepsilon) 205$ (5.98), 225 (0.67), $243(-1.70), 304(-0.51) \mathrm{nm}$. ESIMS $m / z, 595(\mathrm{M}+\mathrm{Na})^{+}$. HRESIMS $m / z, 595.2160$ [calcd for $\left.\mathrm{C}_{30} \mathrm{H}_{36} \mathrm{O}_{11} \mathrm{Na}(\mathrm{M}+\mathrm{Na})^{+}: 595.2155\right]$.

Walsogyne $K(4)$ white amorphous solid. $[\alpha]_{\mathrm{D}}{ }^{28}-12$ (c 0.7, MeOH). IR (film) $v_{\max } 3444,2926,1747,1714$, $1681 \mathrm{~cm}^{-1}$. UV (MeOH) $\lambda_{\max }(\log \varepsilon) 215(4.09) \mathrm{nm} . \mathrm{CD}$ $(\mathrm{MeOH}) \lambda_{\max }(\Delta \varepsilon) 208$ (8.78), $228(-1.41), 247$ (1.77), $296(-0.63), 349(0.29) \mathrm{nm}$. ESIMS $m / z 609(\mathrm{M}+\mathrm{Na})^{+}$. HRESIMS $m / z 609.2309$ [calcd for $\mathrm{C}_{31} \mathrm{H}_{38} \mathrm{O}_{11} \mathrm{Na}(\mathrm{M}+\mathrm{Na})^{+}$: 609.2312].
Walsogyne $L(5)$ white amorphous solid. $[\alpha]_{\mathrm{D}}{ }^{28}-1.5(c$ 0.58, MeOH). IR (film) $v_{\max } 3418,2957,2922,1715 \mathrm{~cm}^{-1}$. $\mathrm{UV}(\mathrm{MeOH}) \lambda_{\max }(\log \varepsilon) 209(4.53) \mathrm{nm} . \mathrm{CD}(\mathrm{MeOH}) \lambda_{\max }$ $(\Delta \varepsilon) 208$ (4.26), 220 (0.30), 242 (2.30), 297 (- 0.55), 350 $(0.29) \mathrm{nm}$. ESIMS $\mathrm{m} / \mathrm{z} 595(\mathrm{M}+\mathrm{Na})^{+}$. HRESIMS $\mathrm{m} / \mathrm{z}$ 595.2183 [calcd for $\mathrm{C}_{30} \mathrm{H}_{36} \mathrm{O}_{11} \mathrm{Na}(\mathrm{M}+\mathrm{Na})^{+}: 595.2155$ ].

Walsogyne $M(6)$ white amorphous solid. $[\alpha]_{\mathrm{D}}{ }^{18}-21$ (c 0.92, MeOH). IR (film) $v_{\max } 3446,2931,1731,1716$, $1698 \mathrm{~cm}^{-1}$. UV $(\mathrm{MeOH}) \lambda_{\max }(\log \varepsilon) 213(4.54) \mathrm{nm} . \mathrm{CD}$ $(\mathrm{MeOH}) \lambda_{\max }(\Delta \varepsilon) 204(9.84), 239(0.038), 296(0.97) \mathrm{nm}$. ESIMS $m / z, 705(\mathrm{M}+\mathrm{Na})^{+}$. HRESIMS $m / z, 705.3121$ [calcd for $\mathrm{C}_{34} \mathrm{H}_{50} \mathrm{O}_{14} \mathrm{Na}(\mathrm{M}+\mathrm{Na})^{+}$: 705.3109].

Walsogyne $N(7)$ white amorphous solid. $[\alpha]_{\mathrm{D}}{ }^{18}-14(c$ 1.00, MeOH). IR (film) $v_{\max } 3393,2919,1716 \mathrm{~cm}^{-1}$. UV $(\mathrm{MeOH}) \lambda_{\max }(\log \varepsilon) 211(4.42) \mathrm{nm} . \mathrm{CD}(\mathrm{MeOH}) \lambda_{\max }(\Delta \varepsilon)$ 209 (4.94), 258 (0.78), $304(-1.34) \mathrm{nm}$. ESIMS $\mathrm{m} / z 619$ $(\mathrm{M}+\mathrm{H})^{+}$. HRESIMS $\mathrm{m} / \mathrm{z} 619.2802\left[(\mathrm{M}+\mathrm{H})^{+}\right.$calcd for $\mathrm{C}_{32} \mathrm{H}_{43} \mathrm{O}_{12}(\mathrm{M}+\mathrm{H})^{+}:$619.2755].

Walsogyne $O(8)$ white amorphous solid. $[\alpha]_{\mathrm{D}}{ }^{18}-32(c$ $0.33, \mathrm{MeOH})$. IR (film) $v_{\max } 3401,2947,1715,1652 \mathrm{~cm}^{-1}$. $\mathrm{UV}(\mathrm{MeOH}) \lambda_{\max }(\log \varepsilon) 216(4.51) \mathrm{nm} . \mathrm{CD}(\mathrm{MeOH}) \lambda_{\max }$ $(\Delta \varepsilon) 210$ (15.32), 240 (-1.04), 287 (-0.50) nm. ESIMS $\mathrm{m} / \mathrm{z} 673(\mathrm{M}+\mathrm{Na})^{+}$. HRESIMS $\mathrm{m} / \mathrm{z} 673.2816$ [calcd for $\mathrm{C}_{33} \mathrm{H}_{46} \mathrm{O}_{13} \mathrm{Na}(\mathrm{M}+\mathrm{Na})^{+}$: 673.2836].

Parasite strain culture P. falciparum laboratory strain 3D7 was obtained from Prof. Masatsugu Kimura (Osaka City University, Osaka, Japan). For the assessment of antimalarial activity of the compounds in vitro, the parasites were cultured in Roswell Park Memorial Institute (RPMI) 1640 medium supplemented with $0.5 \mathrm{~g} / \mathrm{L}$ L-glutamine, $5.96 \mathrm{~g} / \mathrm{L}$ HEPES, $2 \mathrm{~g} / \mathrm{L}$ sodium bicarbonate $\left(\mathrm{NaHCO}_{3}\right)$, $50 \mathrm{mg} / \mathrm{L}$ hypoxanthine, $10 \mathrm{mg} / \mathrm{L}$ gentamicin, $10 \%$ heatinactivated human serum, and red blood cells (RBCs) at a $3 \%$ hematocrit in an atmosphere of $5 \% \mathrm{CO}_{2}, 5 \% \mathrm{O}_{2}$, and $90 \% \mathrm{~N}_{2}$ at $37{ }^{\circ} \mathrm{C}$ as previously described [30]. Ring-form parasites were collected using the sorbitol synchronization technique [31]. Briefly, the cultured parasites were collected by centrifugation at $840 \mathrm{~g}$ for $5 \mathrm{~min}$ at room temperature, suspended in a fivefold volume of 5\% D-sorbitol (Nacalai Tesque, Kyoto, Japan) for $10 \mathrm{~min}$ at room temperature, and then they were washed twice with RPMI 1640 medium to remove the D-sorbitol. The utilization of blood samples of healthy Japanese volunteers for the parasite culture was approved by the institutional review committee of the Research Institute for Microbial Diseases (RIMD), Osaka University (approval number: 22-3).

Antimalarial activity Ring-form-synchronized parasites were cultured with compounds $\mathbf{1 - 8}$ and walsogynes B, D, and $\mathrm{E}$ at sequentially decreasing concentrations $(50,15,5$, $1.5,0.5$, and $0.15 \mu \mathrm{M}$ ) for $48 \mathrm{~h}$ for the flow cytometric analysis using an automated hematology analyzer, XN-30. The $\mathrm{XN}-30$ analyzer was equipped with a prototype algorithm for cultured falciparum parasites [prototype; software version: 
01-03, (build 16)] and used specific reagents (CELLPACK DCL, SULFOLYSER, Lysercell M, and Fluorocell M) (Sysmex, Kobe, Japan) [32, 33]. Approximately $100 \mu \mathrm{L}$ of the culture suspension diluted with $100 \mu \mathrm{L}$ phosphate-buffered saline was added to a BD Microtainer MAP Microtube for Automated Process $\mathrm{K}_{2}$ EDTA $1.0 \mathrm{mg}$ tube (Becton Dickinson and Co., Franklin Lakes, NJ, USA) and loaded onto the $\mathrm{XN}-30$ analyzer with an auto-sampler as described in the instrument manual (Sysmex). The parasitemia (MI-RBC\%) was automatically reported [32]. Then $0.5 \%$ DMSO alone or containing $5 \mu \mathrm{M}$ artemisinin used as the negative and positive controls, respectively. The growth inhibition (GI) rate was calculated from the MI-RBC\% according to the following equation:

$\mathrm{GI}(\%)=100-($ test sample-positive control) $/($ negative control - positive control) $\times 100$

The $\mathrm{IC}_{50}$ was calculated from GI (\%) using GraphPad Prism version 5.0 (GraphPad Prism Software, San Diego, CA, USA) [34].

Supplementary Information The online version contains supplementary material available at https://doi.org/10.1007/s11418-021-01556-4.

Acknowledgements We thank to Prof. Masatsugu Kimura (Osaka City University, Osaka, Japan) for the kind gift of the 3D7 strain, Mr. Yuji Toya and Dr. Kinya Uchihashi (Sysmex) for the setting of the XN-30 analyzer and Ms. Toshie Ishisaka and Ms. Sawako Itagaki for their technical assistance. This work was partly supported by JSPS KAKENHI (JP 19K07152 to MH) and (JP 16K08309 to AEN), Japan.

\section{References}

1. Mabberley DJ (2011) Meliaceae. In: Kubitzki K (ed) Flowering plants Eudicots: Sapindales, Cucurbitales, Myrtaceae. Springer, pp 185-211

2. Mohamad K, Hirasawa Y, Lim CS, Awang K, Hadi AHA, Takeya K, Morita H (2008) Ceramicine A and walsogyne A, novel limonoids from two species of Meliaceae. Tetrahedron Lett 49:4276-4278

3. Nugroho AE, Okuda M, Yamamoto Y, Wong CP, Hirasawa Y, Kaneda T, Shirota O, Hadi AHA, Morita H (2017) Apowalsogynes A and B, two highly oxidized 3,4-seco-apotirucallane triterpenoids from Walsura chrysogyne. Nat Prod Commun 12:1189-1192

4. Nugroho AE, Okuda M, Yamamoto Y, Hirasawa Y, Wong C-P, Kaneda T, Shirota O, Hadi AHA, Morita H (2013) Walsogynes $\mathrm{B}-\mathrm{G}$, limonoids from Walsura chrysogyne. Tetrahedron 69:4139-4145

5. Sichaem J, Siripong P, Tip-pyang S, Phaopongthai J (2014) A new cytotoxic tirucallane from the twigs of Walsura trichostemon. Nat Prod Commun 9:367-368

6. Sichaem J, Aree T, Khumkratok S, Jong-aramruang J, Tip-pyang S (2012) A new cytotoxic apotirucallane from the roots of Walsura trichostemon. Phytochem Lett 5:665-667

7. Mahmod II, Kwong HC, Tahir MIM, Ismail IS (2011) $\left(20 S^{*}, 24 S^{*}\right)$-25-hydroxy-20,24-epoxy-A-homo-4-oxadammaran3-one (Chrysura) isolated from the leaves of Walsura chrysogyne. Acta Crystallogr Sect E 67:03296
8. Kaneda T, Matsumoto M, Sotozono Y, Fukami S, Nugroho AE, Hirasawa Y, Hamid AHA, Morita H (2019) Cycloartane triterpenoid $(23 R, 24 E)$-23-acetoxymangiferonic acid inhibited proliferation and migration in B16-F10 melanoma via MITF downregulation caused by inhibition of both $\beta$-catenin and c-RafMEK1-ERK signaling axis. J Nat Med 73:47-58

9. Nugroho AE, Hashimoto A, Wong C-P, Yokoe H, Tsubuki M, Kaneda T, Hadi AHA, Morita H (2018) Ceramicines M-P from Chisocheton ceramicus: isolation and structure-activity relationship study. J Nat Med 72:64-72

10. Nugroho AE, Inoue D, Wong CP, Hirasawa Y, Kaneda T, Shirota O, Hadi AHA, Morita H (2018) Reinereins A and B, new onocerane triterpenoids from Reinwardtiodendron cinereum. J Nat Med 72:588-592

11. Nugroho AE, Nakamura H, Inoue D, Hirasawa Y, Wong CP, Kaneda T, Hadi AHA, Morita H (2018) Polyisoprenylated acylphloroglucinols from Garcinia nervosa. Nat Prod Commun 13:367-369

12. Nugroho AE, Matsumoto M, Sotozono Y, Kaneda T, Hadi AHA, Morita H (2018) Cycloartane triterpenoids with anti-melanin deposition activity. Nat Prod Commun 13:809-812

13. Nugroho AE, Zhang W, Hirasawa Y, Tang Y, Wong CP, Kaneda T, Hadi AHA, Morita H (2018) Bisleuconothines B-D, modified eburnane-aspidosperma bisindole alkaloids from Leuconotis griffithii. J Nat Prod 81:2600-2604

14. Nugroho AE, Sasaki T, Kaneda T, Hadi AHA, Morita H (2017) Calofolic acids A-F, chromanones from the bark of Calophyllum scriblitifolium with vasorelaxation activity. Bioorg Med Chem Lett 27:2124-2128

15. Iijima C, Wong CP, Nugroho AE, Sotozono Y, Someya S, Hirasawa Y, Kaneda T, Hadi AHA, Morita H (2016) Anti-melanin deposition activity of ceramicines from Chisocheton ceramicus. J Nat Med 70:702-707

16. Nugroho AE, Wong CP, Hirasawa Y, Janar J, Kaneda T, Shirota O, Morita H (2016) Daphnane Diterpenoids from Daphne altaica. Nat Prod Commun 11:1073-1075

17. Nugroho AE, Sugiura R, Momota T, Hirasawa Y, Wong CP, Kaneda T, Hadi AHA, Morita H (2015) Dysosesquiflorins A and B, sesquiterpenoids from Dysoxylum densiflorum. J Nat Med 69:411-415

18. Kiren Y, Nugroho AE, Hirasawa Y, Shirota O, Bekenova M, Narbekovich NO, Shapilova M, Maeno H, Morita H (2014) Mumic acids A-E: new diterpenoids from mumiyo. J Nat Med 68:199-205

19. Prema WCP, Awouafack MD, Nugroho AE, Win YY, Win NN, Ngwe H, Morita H, Morita H (2019) Two new quassinoids and other constituents from the Picrasma javanica wood and their biological activities. J Nat Med 73:589-596

20. Prema WCP, Kodama T, Nugroho AE, El-Desoky AH, Awouafack MD, Win YY, Ngwe H, Abe I, Morita H, Morita H (2020) Three new quassinoids isolated from the wood of Picrasma javanica and their anti-Vpr activities. J Nat Med 74:571-578

21. Nugroho AE, Hirasawa Y, Kaneda T, Shirota O, Matsuno M, Mizukami H, Morita H (2021) Triterpenoids from Walsura trichostemon. J Nat Med 75:415-422

22. Nugroho AE, Ono Y, Jin E, Hirasawa Y, Kaneda T, Rahman A, Kusumawati I, Tougan T, Horii T, Zaini NC, Morita H (2021) Bisindole alkaloids from Voacanga grandifolia leaves. J Nat Med 75:408-414

23. Hirasawa Y, Agawa-Kakimoto M, Zaima K, Uchiyama N, Goda Y, Morita H (2021) Complanadine F, a novel dimeric alkaloid from Lycopodium complanatum. J Nat Med 75:403-407

24. Kaneda T, Nakajima Y, Koshikawa S, Nugroho AE, Morita $\mathrm{H}$ (2019) Cyclolinopeptide F, a cyclic peptide from flaxseed inhibited RANKL-induced osteoclastogenesis via downergulation of RANK expression. J Nat Med 73:504-512 
25. Hirasawa Y, Dai X, Deguchi J, Hatano S, Ohtsuka R, Nugroho AE, Kaneda T, Morita H (2019) New vasorelaxant indole alkaloids, taberniacins A and B, from Tabernaemontana divaricata. $\mathrm{J}$ Nat Med 73:627-632

26. Tang Y, Nugroho AE, Hirasawa Y, Tougan T, Horii T, Hamid A, Hadi A, Morita H (2019) Leucophyllinines A and B, bisindole alkaloids from Leuconotis eugeniifolia. J Nat Med 73:533-540

27. Amelia P, Nugroho AE, Hirasawa Y, Kaneda T, Tougan T, Horii T, Morita H (2019) Indole alkaloids from Tabernaemontana macrocarpa jack. J Nat Med 73:820-825

28. Yin S, Wang XN, Fan CQ, Liao SG, Yue JM (2007) The first limonoid peroxide in the Meliaceae family: walsuronoid A from Walsura robusta. Org Lett 9:2353-2356

29. Mohamad K, Hirasawa Y, Litaudon M, Awang K, Hadi AHA, Takeya K, Ekasari W, Widyawaruyanti A, Zaini NC, Morita H (2009) Ceramicines B-D, new antiplasmodial limonoids from Chisocheton ceramicus. Bioorg Med Chem 17:727-730

30. Trager W, Jensen JB (1976) Human malaria parasites in continuous culture. Science 193:673-675. https://doi.org/10.1126/scien ce.781840

31. Lambros C, Vanderberg JP (1979) Synchronization of Plasmodium falciparum erythrocytic stages in culture. J Parasitol $65: 418-420$
32. Tougan T, Suzuki Y, Itagaki S, Izuka M, Toya Y, Uchihashi K, Horii T (2018) An automated haematology analyzer XN-30 distinguishes developmental stages of falciparum malaria parasite cultured in vitro. Malar J 17:59. https://doi.org/10.1186/ s12936-018-2208-6

33. Toya Y, Tougan T, Horii T, Uchihashi K (2021) Lysercell M enhances the detection of stage-specific Plasmodium-infected red blood cells in the automated hematology analyzer XN-31 prototype. Parasitol Int 80:102206. https://doi.org/10.1016/j.parint. 2020.102206

34. Tougan T, Toya Y, Uchihashi K, Horii T (2019) Application of the automated haematology analyzer XN-30 for discovery and development of anti-malarial drugs. Malar J 18:8. https://doi.org/ 10.1186/s12936-019-2642-0

Publisher's Note Springer Nature remains neutral with regard to jurisdictional claims in published maps and institutional affiliations. 